\title{
Modeling Oil-paper Insulation Frequency Domain Spectroscopy Based on its Microscopic Dielectric Processes
}

\author{
Jiacheng Xie, Ming Dong, Yizhuo Hu, Tianxin Zhuang \\ Ricardo Albarracin-Sánchez, Johnatan M. Rodriguez-Serna
}

\begin{abstract}
The present study sets out to devise a universal function model to explain the characteristic curve obtained in the frequency domain spectroscopy (FDS) test on oilpaper insulation, based on its microscopic conduction and relaxation processes, and thus to enhance the accuracy and applicability of the test. First, from the analysis of the relationship between the real and imaginary parts of the dielectric's complex permittivity, it is demonstrated that a relaxation peak co-exists with the conduction process in the low-frequency band of an FDS curve obtained for oil-impregnated paper sample. Second, values for polarization barrier heights, essential to the determination of the microscopic polarization mechanisms, are presented as results of FDS and thermally stimulated depolarization current experiments carried out on oilimpregnated paper samples. The polarization peaks obtained in the imaginary permittivity frequency spectrum are determined as, respectively, space charge polarization and interface polarization. Finally, a function model in good agreement with experiment data is proposed, which quantitatively describes the FDS curve in oilimpregnated sample, including two relaxation processes and one conduction process.
\end{abstract}

\section{INTRODUCTION}

OIL-PAPER insulation, a classic insulation structure with a long history, has been an excellent solution for the insulation of power equipment and thus continues to attract widespread attention [1]. To ensure the reliable operation of power equipment, as well as enable the formulation of a reasonable maintenance strategy, it is of great importance to accurately evaluate the insulation properties of oil-impregnated paper. Frequency domain spectroscopy (FDS), based on the principle of dielectric response, is one of several techniques with the advantages of strong anti-interference, the provision of richer information of defects, and is non-destructive [2]. This method lends itself to the condition assessment of power equipment.

To guarantee the accuracy and applicability of FDS, many related studies have been carried out to explain the FDS test curve. In addition, studies have been addressed the establishment of qualitative and quantitative relationships between the characteristic parameters of the FDS test and the conditions of the insulation, such as the moisture content and degree of aging $[3,4]$. The insulation's FDS curve essentially is the reflection to the comprehensive effects of material's microscopic charge movements in alternating electric field, namely, polarization and conduction processes. The Debye model, as well as modified versions such as the HavriliakNegami (H-N) formula, has been proposed to quantify the broadband polarization process [5] while Dyre's random freeenergy barrier model describes effectively the conduction process in dielectric materials [6]. In [7], the oil-impregnated paper's FDS curves in the low frequency range $\left(10^{-3}\right.$ to $10^{\circ}$ $\mathrm{Hz}$ ) were shown to be related to conduction losses, while in the high-frequency range $\left(10^{1}\right.$ to $\left.10^{3} \mathrm{~Hz}\right)$, relaxation losses are dominant. Related works have developed function models taking into account the conduction and polarization processes, to explain the behavior of the FDS test curve in the entire test frequency band as described above [8].

However, the specific physical mechanisms of oil-paper insulation's FDS curve are still controversial. Recent 
researches in the area of dielectric physics have revealed that, for several dielectric materials, there are more factors for dielectric losses under low-frequency voltages than what conventionally believed to be only generated by the conduction processes. Tian and Ohki, using the concept of electric modulus, demonstrated that a low-frequency relaxation process overlapped with conduction process exists in the response spectra of various polymeric materials (epoxy resin, low-density polyethylene, polyethylene terephthalate and liquid-crystal polymers) [9]. In addition, it has been proved that a space charge polarization with a rather long relaxation time co-exists with high conductance losses in $\mathrm{CaCu}_{3} \mathrm{Ti}_{4} \mathrm{O}_{12}$ (CCTO) [10]. These research progresses are with reference values to clarify the principles of oil-impregnated paper's FDS curve at the micro-level, particularly in the lowfrequency band.

Being limited by the lack of the related basic theories and evaluation criteria with precision and universality, FDS test is currently used only as an auxiliary tool in the diagnosis field. The main objective of the present study is to clarify all the conduction and polarization processes in frequency domain, determine their microscopic mechanisms, then separate each process from the overlapped spectrum and eventually model the FDS curve. In Section 2 of this paper, basic theories of material's dielectric response are introduced, in which a mathematical equation is deduced to separate polarization losses when they overlap with conduction losses. Section 3 of this paper illustrates the experimental setups, where FDS and thermally stimulated depolarization current (TSDC) are jointly carried out on prepared oil-impregnated paper samples. Analysis and discussion based on experimental data are presented in Section 4, whereby a function model of the oilimpregnated paper's FDS curve is derived after its microscope dielectric processes are ascertained. An accurate function model of the FDS not only is capable to provide sufficient characteristic parameters related to the conditions of oilimpregnated paper insulation such as moisture or aging degree, but also allows FDS to be a more reliable method for field insulation diagnosis of power transformers and other capacitive equipment. With the above-mentioned merits, this model could contribute to the urgent demands of electric power reliability.

\section{THEORY}

\subsection{RELATIONSHIP BETWEEN REAL AND IMAGINARY PARTS OF COMPLEX PERMITTIVITY}

In an applied alternating electric field, the charged particles' transfer processes and a surface binding effect exist concurrently in a dielectric material, which lead to the conduction and polarization processes, respectively. With the variation in the frequency of the applied voltage, there are changes in the phase and amplitude of the output current in the dielectric material due to the different responses of the microscopic polarization and conduction processes in frequency domain [5]. Based on this, the definition of complex capacitance and the complex permittivity, functions of angular frequency $\omega$ reflecting the conduction and polarization of charges at the macro level, can be expressed by Equation (1) [8]:

$$
I^{*}(\omega) / U^{*}(\omega)=i \omega\left[C^{\prime}(\omega)-i C^{\prime \prime}(\omega)\right]=i \omega C_{0}\left[\varepsilon^{\prime}(\omega)-i \varepsilon^{\prime \prime}(\omega)\right]
$$

where $I^{*}(\omega)$ and $U^{*}(\omega)$ are tested current and applied voltage in complex number form, $C^{\prime}(\omega)$ and $C^{\prime \prime}(\omega)$ the real and imaginary parts of the complex capacitance respectively; $C_{0}$ stands for the vacuum capacitance, while $\varepsilon^{\prime}(\omega)$ and $\varepsilon^{\prime \prime}(\omega)$ are, respectively, the real and imaginary parts of the complex permittivity. Additionally, $\varepsilon^{\prime}(\omega)$ reflects the charge storage capacity of the dielectric while $\varepsilon "(\omega)$ is related to the dielectric losses [11], to be specific, conductance and polarization losses.

For the conduction process, Dyre effectively demonstrated that dielectric losses in solid dielectrics generated by DC conductance depend on particles' detrapping processes, based on which the charge random free-energy barrier model was developed [6]. Further deduction on the effect of conductance in the complex permittivity of dielectric material $\varepsilon^{*}$, according to the above model, could be determined through Equation (2), which is widely accepted in the literature [12].

$\varepsilon_{D C}^{*}=\sigma_{D C} /\left(i \omega \varepsilon_{0}\right)$

where $\sigma_{D C}$ is the DC conductivity of the material and $\varepsilon_{0}$ is the vacuum permittivity with a value of $8.85 \times 10^{-12} \mathrm{~F} / \mathrm{m}$. For polymers and amorphous materials, the barrier-hopping movement of electrons is the main cause of their conduction processes [13]. With the effect of an applied electric field, due to the directional thermal motion, electrons continuously cross the trap barriers and then pass through the sample to be eventually neutralized by the electrodes. This process is greatly weakened when the frequency of the alternating electric field increases. Equation (2) corresponds to the function model, which is linear in logarithmic coordinates with a slope of -1 [13].

The Debye function is one of the most recognized models for analyzing the polarization process with exact physical implications [4]. Equation (3) gives the function model for the polarization process

$\varepsilon_{p}^{*}(\omega)=\varepsilon_{\infty}+\left(\varepsilon_{s}-\varepsilon_{\infty}\right) /(1+i \omega \tau)$

where, $\varepsilon_{s}$ and $\varepsilon_{\infty}$ are, respectively, the permittivities at low and high frequencies for a single relaxation process, and $\tau$ is the polarization time. According to Equation (3), the functions of $\varepsilon$ ' and $\varepsilon$ " could be obtained individually, while their quantitative relationship turns to be a semicircle in coordinates, known as the Debye semicircle. In the past few decades, hundreds of spectra in dielectric materials have been determined, with some exhibiting obvious deviations between the Debye semicircle and the experimental data [14]. Thus, modified models such as the Cole-Cole model and the H-N function have been developed by adding correction factors based on the Debye model. Equation (4) corresponds to the H$\mathrm{N}$ model [5]

$\varepsilon_{H N}^{*}(\omega)=\varepsilon_{\infty}+\Delta \varepsilon /\left(1+\left(i \omega \tau_{\mathrm{HN}}\right)^{\alpha}\right)^{\beta}$ 
where $\Delta \varepsilon$ is the difference between $\varepsilon_{S}$ and $\varepsilon_{\infty}, \tau_{H N}$ is the polarization time constant obeying the $\mathrm{H}-\mathrm{N}$ function [15] and $\alpha$ and $\beta$ are coefficients related to sample's structure and shape.

Figure 1 presents a comprehensive description of each parameter in the Debye model, as well as the H-N function model for a single polarization process. It is clear that, for a single relaxation process without the participation of charge conduction, there is a one-to-one correspondence between the real and imaginary parts of the complex permittivity in both, the Debye and the $\mathrm{H}-\mathrm{N}$ models. The appearance of a relaxation loss peak in the frequency spectrum of the imaginary part of permittivity is always accompanied by a decline in the real part spectrum. To explain this regulation at the macro level, according to the introduction above, $\varepsilon$ ' reflects the ability of the dielectric material to resist an applied electric field, which enables the electrodes to produce additional induced electric charges on the surface to eliminate this resistance. Thus, when the frequency of the applied voltage is close to the characteristic frequency of relaxation, the polarization particles will continue to move or be oriented during the entire voltage period, which will lead to significant losses and produce a relaxation peak in the spectrum of $\varepsilon$ ". Meanwhile, with the increasing in frequency, as the polarization partials fail to keep pace with the alternating voltage, the relaxation's electric field resistance effect will decrease significantly, leading to a decline in the spectrum of $\varepsilon$ '.

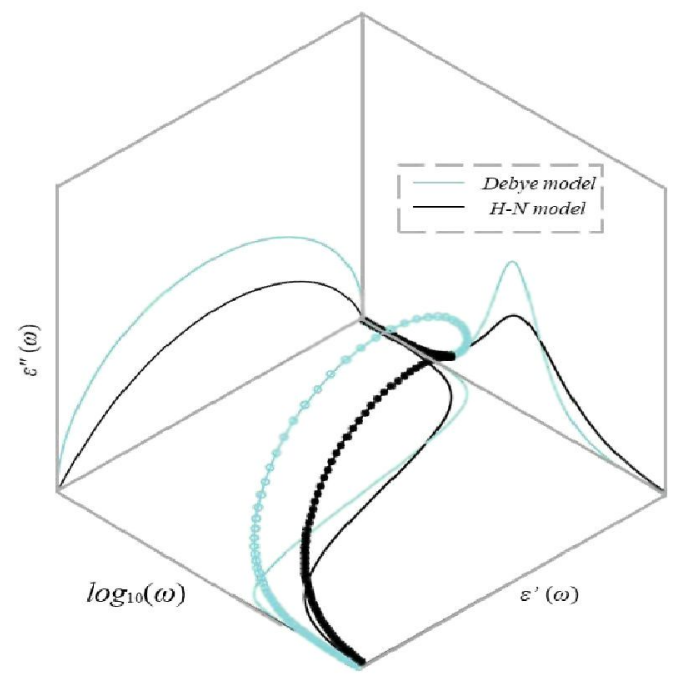

Figure 1. Relationships between $\varepsilon^{\prime}, \varepsilon^{\prime \prime}$ and testing frequency for a single relaxation process described by Debye and $\mathrm{H}-\mathrm{N}$ models.

However, the actual dielectric spectrum obtained by FDS test is a comprehensive effect of polarization and conduction, as deduced from Equation (5)

$\varepsilon^{*}(\omega)=\varepsilon_{p}^{*}(\omega)+\varepsilon_{d}^{*}(\omega)=\varepsilon_{\infty}+\left(\varepsilon_{s}-\varepsilon_{\infty}\right) /(1+i \omega \tau)+\sigma_{0} /\left(i \varepsilon_{0} \omega\right)$

where $\varepsilon_{p}$ and $\varepsilon_{d}$ indicate, respectively, the permittivities caused by the polarization and conduction under broadband applied voltage, while $\sigma_{0}$ is the material's DC conductivity. Meanwhile, the relationship between permittivity's real part $\varepsilon$ ' and imaginary part $\varepsilon "$, which is determined to be a Debye semicircle, is not applicable in this frequency range.

\subsection{DEDUCTION OF LOW-FREQUENCY POLARIZATION QUANTIFYING FACTOR}

The low-frequency range of the $\varepsilon$ " spectrum in oilimpregnated paper samples, as tested by FDS and in logarithmic coordinates, reveals an approximate linear decreasing. Much of the related literature has concluded that the low-frequency band of the FDS test curve is dominated by the conductance [16]. According to their test data [17], however, there has always been an obvious decline in the value of $\varepsilon$ ' with an increase in the frequency, indicating that a relaxation process may exist, although this is concealed by conduction losses. Based on the analysis given above, the existence of conduction process leads to the inconsistency between test data in the low-frequency range and Debye semicircle, which is merely related to the polarization process. Such inconformity makes Debye semicircle an effective tool to identify the polarization component in $\varepsilon$ " by $\varepsilon^{\prime}$.

Based on Equation (3), the functions for $\varepsilon$ ' and $\varepsilon_{p}$ " could be expressed by Equation (6) and Equation (7)

$\varepsilon^{\prime}=\varepsilon_{\infty}+\left(\varepsilon_{\mathrm{s}}-\varepsilon_{\infty}\right) /\left(1+(\omega \tau)^{2}\right)$

$\varepsilon_{p}{ }^{\prime \prime}=\left(\varepsilon_{\mathrm{s}}-\varepsilon_{\infty}\right) \omega \tau /\left(1+(\omega \tau)^{2}\right)$

Here, $\varepsilon_{p}$ " stands for the imaginary part of $\varepsilon_{p}$ and is related to the polarization losses. Based on Equations (6) and (7), a quantitative relationship between $\varepsilon^{\prime}$ and $\varepsilon_{p}$ " is proposed, as given by Equation (8)

$$
\begin{aligned}
-\mathrm{d} \varepsilon^{\prime} / \mathrm{d}\left(\log _{10} \omega\right) & =-\mathrm{d}\left[\varepsilon_{\infty}+\left(\varepsilon_{\mathrm{s}}-\varepsilon_{\infty}\right) /\left(1+(\omega \tau)^{2}\right)\right] / \mathrm{d} \log _{10} \omega \\
& =-\mathrm{d}\left[\varepsilon_{\infty}+\left(\varepsilon_{\mathrm{s}}-\varepsilon_{\infty}\right) /\left(1+\tau^{2} 10^{2 \log _{10} \omega}\right)\right] / \mathrm{d}_{\log } \omega \\
& =2 \ln 10 /\left(\varepsilon_{\mathrm{s}}-\varepsilon_{\infty}\right) \cdot\left[\left(\varepsilon_{\mathrm{s}}-\varepsilon_{\infty}\right) \omega \tau /\left(1+(\omega \tau)^{2}\right)\right]^{2} \\
& =K \cdot\left(\varepsilon_{p}\right)^{2}
\end{aligned}
$$

where $K$, representing the formula $2 \ln 10 /\left(\varepsilon_{s}-\varepsilon_{\infty}\right)$, is an exact value that can be calculated by experimental data.

Given that value of $K$ always remains positive, the rightmost side of Equation (8) indicates that polarization loss has a specific positive correlation with the formula $\mathrm{d} \varepsilon^{\prime} / \mathrm{d}\left(\log _{10} \omega\right)$. Thus, as long as a dielectric material's frequency response spectrum is obtained in logarithmic coordinates, the leftmost side of Equation (8) will turn to be an effective means for revealing the losses caused only by the polarization process in the frequency range where the conduction and polarization overlap.

\section{EXPERIMENTAL SETUP}

\subsection{SAMPLE PREPARATION}

Pressboards of $2 \mathrm{~mm}$ in thickness, produced by Weidmann Co., Ltd., and ordinary \#25 insulating oil were selected to prepare the oil-impregnated paper samples for the tests. The preparation was done in accordance with IEC 60641-2 standard [18]. Before the immersion process, the pressboard and oil were separately placed in an oven at $105^{\circ} \mathrm{C}$ for $24 \mathrm{~h}$ to remove any water. The Karl Fischer method was used to 
determine the moisture content of the raw materials and the drying process was only terminated when the moisture content had fallen below $0.5 \%$. Then, the dried pressboard samples were immersed in the insulating oil at vacuum for $48 \mathrm{~h}$ to guarantee the adequacy of immersion. The prepared oilimpregnated paper samples were kept sealed in a special container to prevent the ingress of moisture.

\subsection{FDS MEASUREMENT}

To implement the FDS tests, the insulation diagnostic analyzer IDAX-300 (Megger Co., Ltd.), widely used device in transformer field assessment, was selected. An experimental chamber was specially designed with three copper electrodes in order to eliminate the influence of the surface-leakage current of the sample. The experimental setup is shown in Figure 2 .

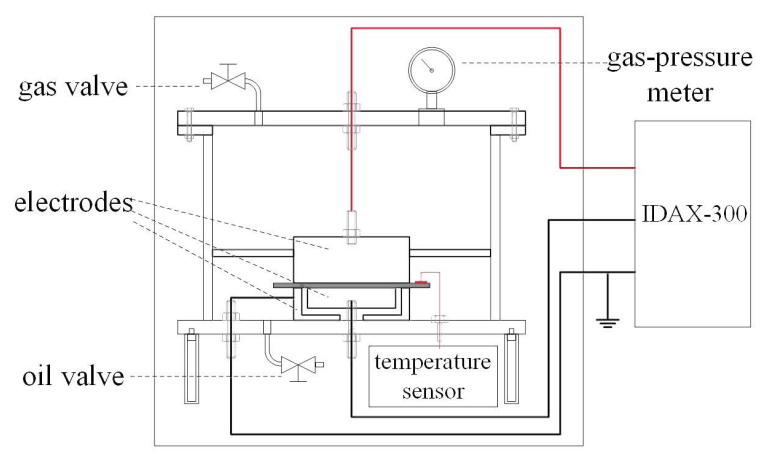

Figure 2. Sketch of FDS experimental device.

A series of sinusoidal waves with peak values of $200 \mathrm{~V}$ and frequencies in the $10^{-3}-10^{5} \mathrm{~Hz}$ range were applied. To guarantee the accuracy of the data processing, 20 testing points were equidistantly fetched in each unit interval in logarithmic coordinates. The samples were immersed in insulating oil at vacuum throughout the test.

To determine the effect of temperature on the polarization process, the FDS curves of the oil-impregnated pressboard samples were also measured at different temperatures. The experimental temperatures were set to $55,60,65,70,75,80$, 85 , and $90^{\circ} \mathrm{C}$.

\subsection{TSDC TEST}

In the present study, the TSDC test was used as an auxiliary test for obtaining the barrier heights of polarization processes in order to ascertain the microscopic polarization mechanisms.

During the TSDC measurements, a DC polarization voltage with an amplitude of $250 \mathrm{~V}$ was applied at a constant temperature of $30{ }^{\circ} \mathrm{C}$ for $40 \mathrm{~min}$ to ensure that various polarizations were attained. Then, the temperature was linearly decreased to $-100{ }^{\circ} \mathrm{C}$ to freeze the relaxation particles. The selection of the minimum temperature was based on preexperiment results and those presented in related literature $[19$, 22]. Subsequently, the temperature was increased at a heating rate of $3{ }^{\circ} \mathrm{C} / \mathrm{min}$ during the depolarization stage after removing the applied voltage. The trend curves of the applied temperature and voltage are shown in Figure 3.

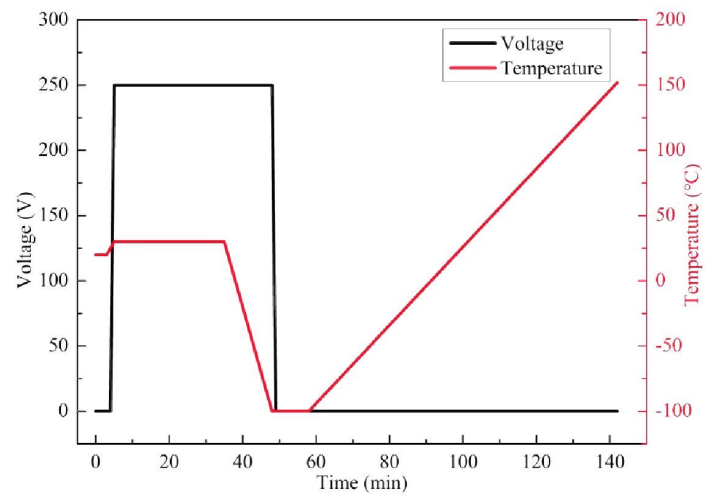

Figure 3. Applied temperature and voltage's trend curve with time.

According to Wang [19], by applying additional bias voltages during the heating stage of the TSDC test, the trend in the variation in the charge quantity is adequate for studying the microscopic mechanism of each polarization process. Thus, in this study, bias voltages of $-100,-50,50$, and $100 \mathrm{~V}$ was applied for $60 \mathrm{~min}$ after the start of the heating stage in the TSDC tests.

An overview of the test process is shown in Figure 4.

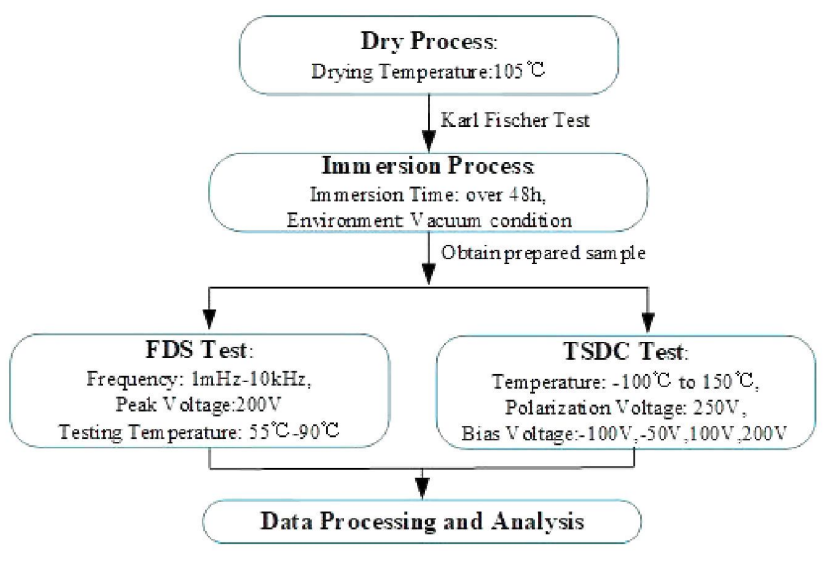

Figure 4. Flow of the testing process.

\section{DATA PROCESSING AND DISCUSSION}

\subsection{MICROSCOPIC DIELECTRIC PROCESSES IN OIL-IMPREGNATED PAPER IN THE FREQUENCY DOMAIN}

After the FDS curves of prepared samples at different temperatures were obtained, a data processing procedure was carried out to determine the microscopic dielectric processes in frequency domain and, especially, to verify the existence of the assumed low-frequency range polarization. This is illustrated in Figure 5.

Figure 5 shows the dielectric spectra of the real and imaginary parts of the permittivity and the quantitative relationship calculated by the leftmost side of Equation (8), at a testing temperature of $60{ }^{\circ} \mathrm{C}$ for an oil-impregnated paper sample. This temperature was found to be appropriate for revealing all the microscopic dielectric processes, based on experimental data acquired for this study. Besides, Figure 5 
clearly illustrates that the $\varepsilon$ " spectrum, obtained by experiment, does not reflect the correspondence with the decline in the $\varepsilon$ ' spectrum when the testing frequency is less than $10^{0} \mathrm{~Hz}$ for relatively large DC conduction losses. On the other hand, the quantitative relationship calculated with Equation (8) eliminates the effect of conductance losses and clearly reveals a low-frequency relaxation with a peak shape. The occurrence of this relaxation not only confirms the validity of above assumption, but also unveils a previously unknown phenomenon that has neither been observed nor studied in the field of frequency spectrum analysis of oil-impregnated paper samples. In addition, another polarization process with a relatively high peak frequency of about $10^{5} \mathrm{~Hz}$ can be easily observed in the $\varepsilon$ " spectrum. Those two relaxation polarization peaks are indicated as peak a and peak $b$.

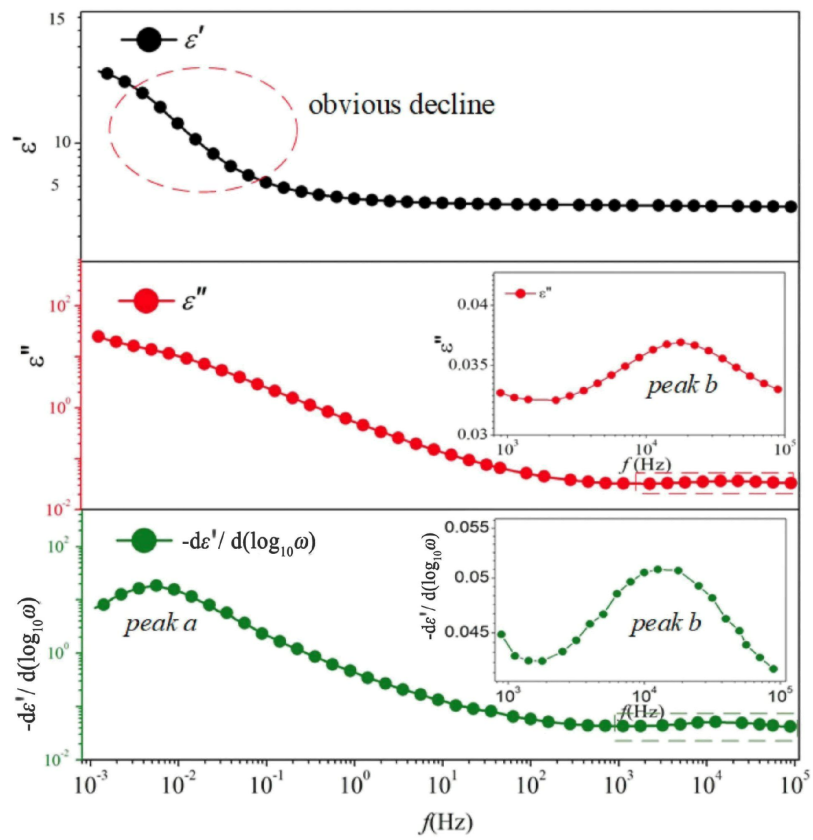

Figure 5. Oil-impregnated paper sample's FDS tested data and calculated results of $-\mathrm{d} \varepsilon^{\prime} / \mathrm{d}\left(\log _{10} \omega\right)$ at $60^{\circ} \mathrm{C}$.

\subsection{GENERATION MECHANISM OF EACH DIELECTRIC PROCESS}

The distribution of charged particles participating in the relaxation process accords with the Boltzmann relationship [20], as defined by Equation (9)

$f_{\text {peak }}=\omega_{0} / \pi \cdot \exp (-H / k T)$

where $f_{\text {peak }}$ is the frequency of relaxation peak, $H$ is the barrier height in $\mathrm{eV}, \omega_{0}$ is the intrinsic angular frequency of captured polarization particles, $k$ is the Boltzmann constant with a value of $1.3806505 \times 10^{-23} \mathrm{~J} / \mathrm{K}$, and $T$ is the temperature in $\mathrm{K}$.

Equation (9) indicates that the Boltzmann distribution is highly temperature-dependent.

The dielectric spectra of peak $a$ and peak $b$ in Figure 5, are shown in Figure 6 for different temperatures. In Figure 6, the barrier heights (or activation energies), can be calculated by using the linear relationship between $H$ and $f_{\text {peak }}$ in logarithmic coordinates according to Equation (9). For peak $a$ and peak $b$, their barrier heights are, respectively, 1.07 and $0.56 \mathrm{eV}$.

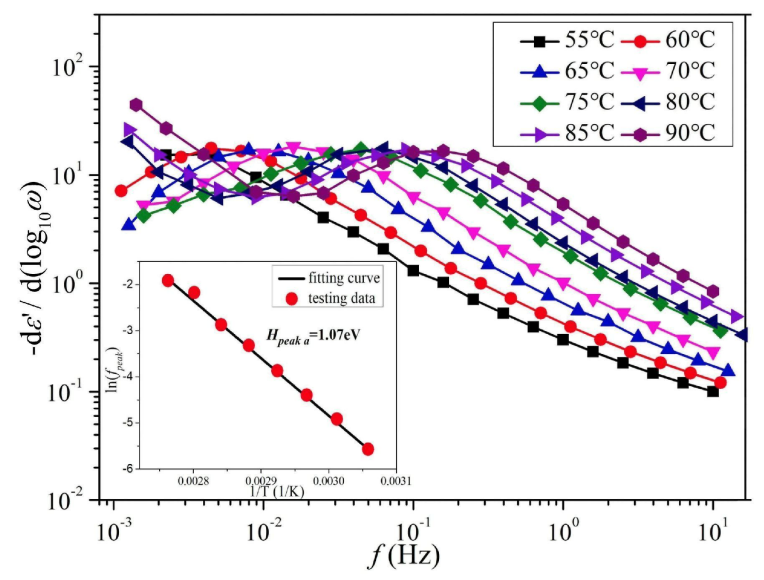

(a) Spectra of the $-\mathrm{d} \varepsilon^{\prime} / \mathrm{d}\left(\log _{10} \omega\right)$ calculated results of peak a.

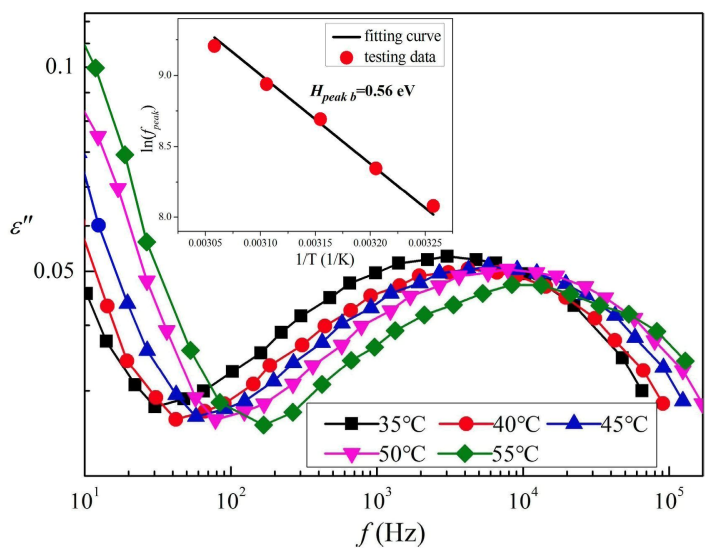

(b) Spectra of the tested $\varepsilon$ ' of peak $b$

Figure 6. Temperature characteristics of peak $a$ and peak $b$ and their fitted polarization barrier heights.

Additionally, in Figure 6a, when the experimental temperature is higher than $80{ }^{\circ} \mathrm{C}$, the results calculated with formula $-\mathrm{d} \varepsilon / \mathrm{d}\left(\log _{10} \omega\right)$ exhibit a descending trend before peak $a$ appears, which signals a clear difference from the curves obtained at other temperatures. This phenomenon, known as low-frequency dispersion, mainly occurs at around $10^{-6} \mathrm{~Hz}$ and at room temperature [21], which is much lower than the FDS test band and thus is not a focus in this paper. However, its occurrence indicates that the quantitative relationship proposed here, in Equation (8), is also sensitive to this phenomenon.

The solid line in Figure 7 shows the experimental TSDC results, for which three different peaks could be observed in the temperature spectroscopy, indicating that there are three different types of polarization in the oil-impregnated paper. These are recorded as, respectively, peak $\alpha$, peak $\beta$, and peak $\gamma$. Moreover, Figure 7 further presents the peak-splitting results based on the expression of thermally stimulated current, as defined by Equation (10) [22]

$I(T)=p_{m} / \tau \cdot \exp (-H /(k T)) \exp \left[-1 /(\tau \beta) \int_{T_{0}}^{T} \exp (-H /(k T)) d T\right]$ 
where $p_{m}$ is the polarization intensity, $\tau$ is the relaxation time and $\beta$ is an undetermined coefficient.

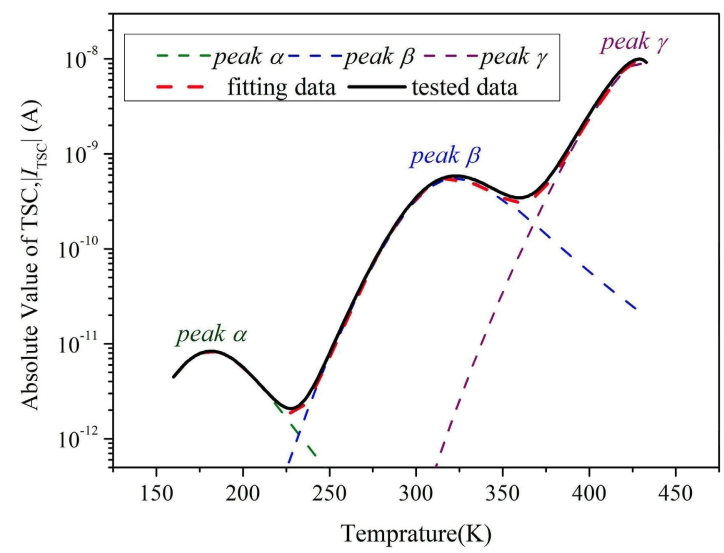

Figure 7. Absolute values of sample's TSDC testing data, fitting curve of each polarization peak and the fitting result of entire TSDC curve.

With Equation (10), the barrier heights of the three polarization peaks could be easily calculated. The results are listed in Table 1, which conform to the values reported by Zhu [22].

Table 1. Fitting function of relaxation's barrier height from TSDC.

\begin{tabular}{c|c|c|c}
\hline Peak & $\alpha$ & $\beta$ & $\gamma$ \\
\hline $\begin{array}{c}\text { Barrier } \\
\text { Height/eV }\end{array}$ & 0.17 & 0.54 & 1.03 \\
\hline
\end{tabular}

Obviously, there is a good consistency between the barrier heights calculated via FDS and the TSDC test results, indicating that peak $a$ in Figure 6(a) describes the same relaxation process as peak $\gamma$ with a barrier height of about $1.03 \mathrm{eV}$, while peak $b$ in Figure $6 \mathrm{~b}$ corresponds to peak $\beta$, of which the barrier height is $0.54 \mathrm{eV}$.

According to Wang [19], for space charge polarization, the charge quantity varies linearly with the bias voltage and becomes negative when the polarity of bias voltage changes. However, for the dipole polarization, the charge quantity is always positive and proportional to the absolute value of the bias voltage. The calculated charge quantity versus the bias voltage is shown in Figure 8, from which it can be seen that peak $a$ in the FDS test curve, as well as peak $\gamma$ in the TSDC test curve of an oil-impregnated paper sample, is a typical polarization relaxation that is generated by the accumulated space charges captured by the traps. Moreover, peak $\alpha$ in the TSDC test curve is the result of a dipole polarization, but with the limitation of the measurement bandwidth, this type of polarization cannot be reflected by the $\varepsilon$ "spectrum as a relaxation peak form. For peak $\beta$, its calculated charge quantity also always stays positive and, unlike peak $\alpha$, it remains constant as the bias voltage varies. This phenomenon has been widely investigated for several kinds of compound insulation samples and is inferred to be generated at the interface between different materials [19].

The above allows to state that the FDS test curve of an oilimpregnated paper sample exhibits three different kinds of dielectric processes, namely, a conduction and a space charge polarization which co-exist in the low-frequency range, as well as an interfacial polarization in the high-frequency range.

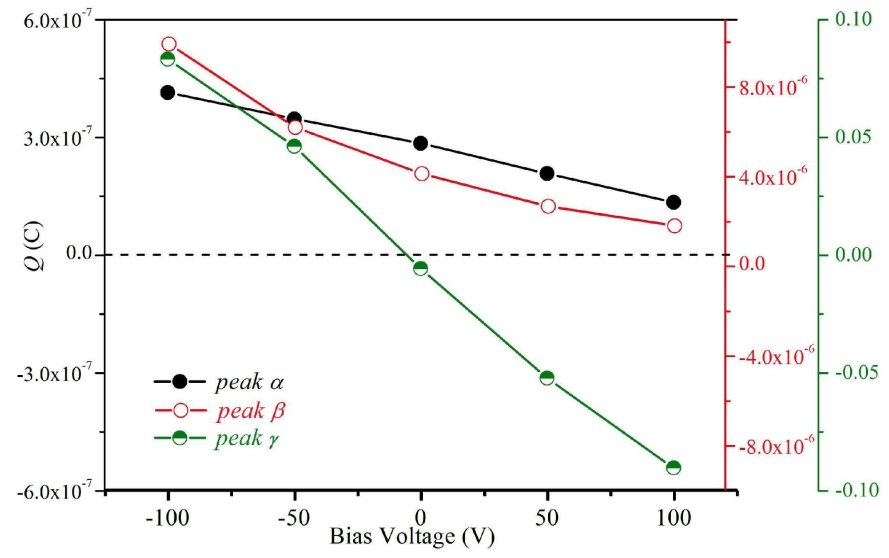

Figure 8. Charge quantity of each polarization peak tested by TSDC under series of bias voltages.

At the microscopic scale, the space charge polarization is mainly triggered by the binding effect on the surfaces of dielectric materials and the electrodes. The accumulation and dissipation processes of space charge are time-consuming, and several minutes or even hours are required for producing obvious dielectric losses. Therefore, its characteristic frequency is rather low. Meanwhile, the interfacial polarization stems from the loose structure of the insulation paper and is assumed to become significant when the insulation material gradually ages and deteriorates. As mentioned above, the conduction process in a dielectric material under an alternating electric field is generated by the barrier-hopping movements of electrons, and the related losses can be calculated with Equation (2), which displays a linear drop of losses against an increase of frequency.

\subsection{QUANTITATIVE MODELING OF FDS TEST CURVE IN OIL-IMPREGNATED PAPER}

After all the dielectric processes, as well as their microscopic mechanisms, are comfirmed, a universal explanation model for the FDS test curve in an oilimpregnated paper sample can be established, by separately quantifying those three processes, as described in Section 4.1, and then recombining them. In the present study, the modeling of the $\varepsilon "(\omega)$ is the focus for its effectiveness in describing both the conductance and polarization losses.

To quantify the $\varepsilon$ "' $(\omega)$ spectrum in the low-frequency range where conductance loss and polarization loss overlap, it is the actual value of polarization loss, namely, $\varepsilon_{p}$ " rather than the formula $-\mathrm{d} \varepsilon^{\prime} / \mathrm{d}\left(\log _{10} \omega\right)$ that is of great necessity. According to Equation (8), the calculation of $\varepsilon_{p}$ " depends on parameters $\varepsilon_{s}$ and $\varepsilon_{\infty}$ of the space charge polarization, which can be directly obtained from FDS experiment data. Meanwhile, limited by FDS measurement bandwidth, the acquisition of $\varepsilon_{s}$ calls for relatively high temperatures at which a polarization peak appears at higher frequencies. The calculation of $\varepsilon_{p}$ " is shown in Equation (11), which obviously is a deformation of 
Equation (8). Then, by taking the total losses, namely, the measured data of $\varepsilon$ ", to substrate the calculated polarization losses, $\varepsilon_{p}$ ", the differences turn to be conductance losses, which are supposed to be linear in logarithmic coordinates and correspond to Equation (2). Then, the overlapped two kinds of different dielectric losses are effectively separated and independently fitted. As for the interfacial polarization in relatively high frequency range, the data can be fitted directly for the conductance losses are negligible

$\varepsilon_{p}^{\prime \prime}=\sqrt{\frac{\left(-\partial \varepsilon^{\prime} / \partial \log _{10} \omega\right)}{2 \ln 10 /\left(\varepsilon_{\mathrm{s}}-\varepsilon_{\infty}\right)}}$

Based on the description above, the FDS curve at $75^{\circ} \mathrm{C}$, of which the temperature is high enough to give $\varepsilon_{s}$ of the space charge polarization, is selected as an appropriate example, to illustrate each modeling step, as shown in Figure 9.

In Figure 9a, the space charge polarization losses are calculated by the quantitative relationship given by Equation (8). The DC conduction losses are obtained by directly subtracting the polarization losses from the measured $\varepsilon$ " spectrum in the low-frequency range. The result turns out to be a perfect line in the spectrum. Further, a fitting procedure according to Equation (2) based on the principle of least square method reveals that the slope of the linear region of conduction losses is equal to -0.9975 , as given by Equation (12), which is very close to -1 , the theoretical value. This fitting result, with a goodness-of-fit of $99.64 \%$, clearly confirms the efficacy of Equation (8) for investigating the concealed polarization losses when they co-exist with large conduction losses

$\log _{10}\left(\varepsilon_{\sigma}{ }^{\prime \prime}\right)=-0.65974-0.99657 \log _{10}(f)$

To enhance the accuracy of the fitting result, the modified Debye model was utilized to describe the polarization processes. Figure $9 \mathrm{~b}$ shows the fitting curve for the space charge polarization according to $\mathrm{H}-\mathrm{N}$ function described by Equation (4), which owns more undetermined coefficients than the Debye model to enhance the coincidence degree of the fitting results. The function model of the curve shown in Figure $9 \mathrm{~b}$ is given by Equation (13), of which the coefficient of determination $\mathrm{R}^{2}$ is equal to 0.9932 . Considering the inherent error of the fitting process, for fitted $\alpha$ and $\beta$ are closed to 1 , the space charge polarization approximately obeys the Debye model

$\varepsilon_{p 1}{ }^{\prime \prime}(f)=\operatorname{Im}\left[1.173 /\left(1+(i 86.079 f)^{0.99763}\right)^{0.97258}\right]$

where, $\operatorname{Im}[]$ stands for the imaginary part of a complex number.

The Equation (14), defining an $\mathrm{H}-\mathrm{N}$ relaxation process, shows the fitting function of the interfacial polarization of oilimpregnated paper samples in the high-frequency range, and its coefficient of determination turns to be 0.988 . Figure 9c further displays the fitting effect. Limited by the measurement bandwidth, the relaxation peak of the interfacial polarization is not apparent in the spectrum at $75{ }^{\circ} \mathrm{C}$, which should be at around $8 \times 10^{4} \mathrm{~Hz}$

$\varepsilon_{p 2}{ }^{\prime \prime}(f)=\operatorname{Im}\left[5.7131 /\left(1+\left(i 8.2228 \times 10^{-5} f\right)^{0.261}\right)^{0.0284}\right]$

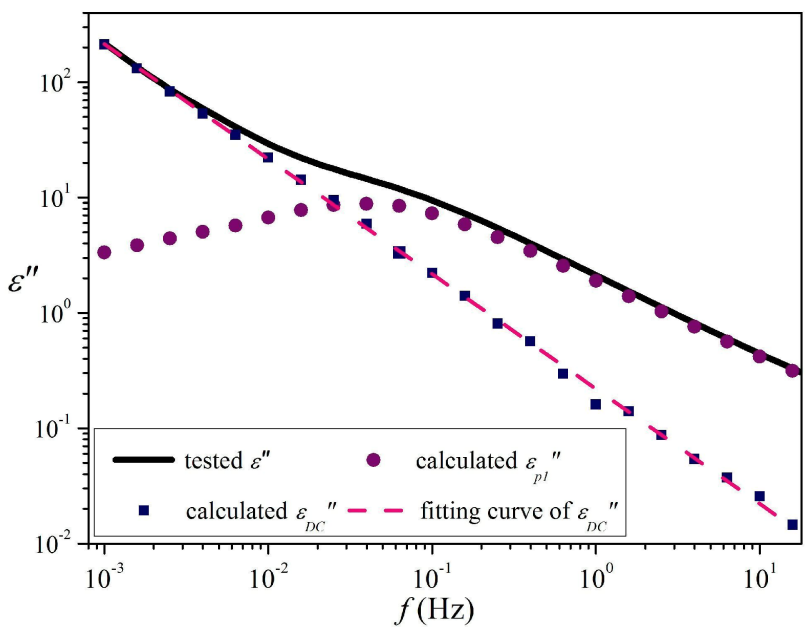

(a) Step 1: Acquisition and fitting process of conductance losses.

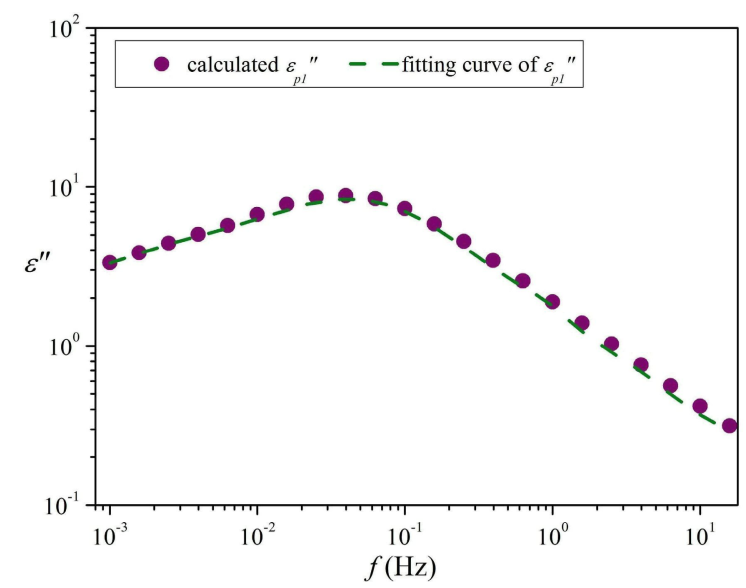

(b) Step 2: Fitting of the space charge polarization process, which approximates to the Debye model.

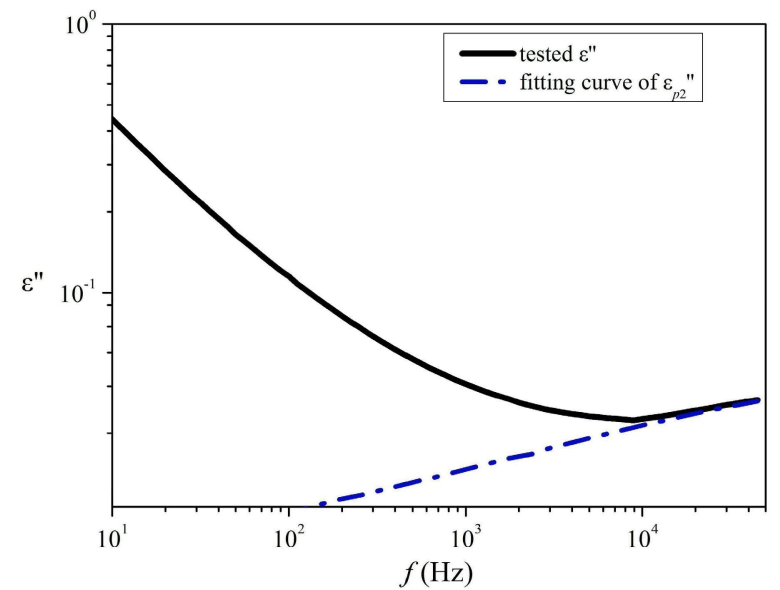

(c) Step 3: Fitting of the interfacial polarization process according to $\mathrm{H}-\mathrm{N}$ model

Figure 9. Modeling methods for each single dielectric process.

After the function model for each individual dielectric process in the test frequency range is obtained, the complete FDS test curve can be drawn by combining the function models of all the microscopic dielectric processes in both, low- and high-frequency ranges. As shown in Figure 10, the fitting result based on the response of the dielectric 
microscopic mechanisms is in good agreement with the measured data. The overall goodness-of-fit is $99.23 \%$ in this case.

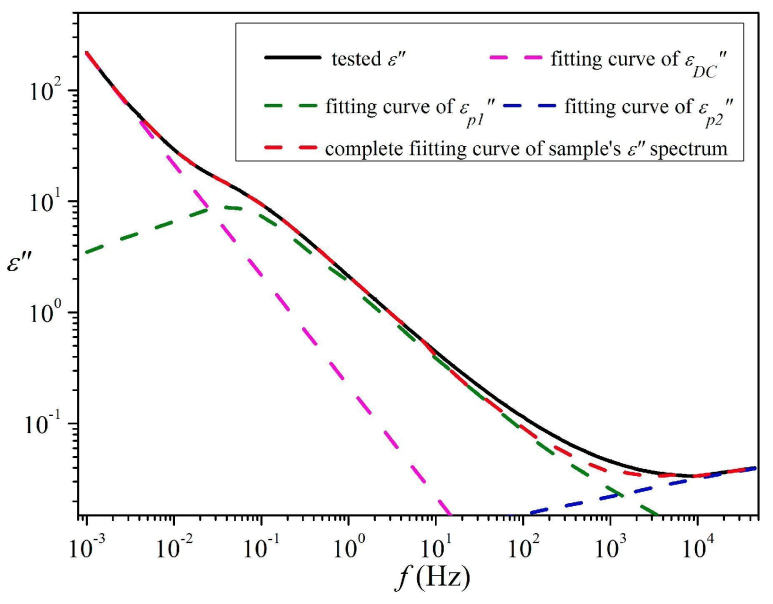

Figure 10. The modeling processes for each single dielectric behavior.

A more universal function model of the imaginary permittivity in oil-impregnated paper samples, based on its dielectric processes in the frequency domain, is defined by Equation (15), according to the analysis made above

$$
\begin{aligned}
\varepsilon^{\prime \prime}(\omega)= & \sigma_{D c} / i \omega \varepsilon_{0} \\
& +\operatorname{Im}\left(\varepsilon_{s 1}-\varepsilon_{\infty 1} / 1+i \omega \tau_{1}\right) \\
& +\operatorname{Im}\left[\varepsilon_{\infty 2}+\left(\varepsilon_{s 2}-\varepsilon_{\infty 2}\right) /\left(1+\left(i \omega \tau_{2}\right)^{\alpha}\right)^{\beta}\right]
\end{aligned}
$$

where, $\varepsilon_{s 1}$ and $\varepsilon_{s 2}$ are the permittivities of space charge polarization and interface polarization at low frequencies while $\varepsilon_{\infty 1}$ and $\varepsilon_{\infty 2}$ are the counterparts at high frequencies, besides, $\tau_{1}$ and $\tau_{2}$ are relaxation time constants of space charge polarization and interface polarization, respectively.

Equation (15), a universal function for oil-impregnated paper's FDS curve, effectively provides characteristic parameters such as $\sigma_{\mathrm{DC}}, \varepsilon_{s l}, \varepsilon_{s 2}, \varepsilon_{\infty 1}, \varepsilon_{\infty 2}, \tau_{1}, \tau_{2}, \alpha$ and $\beta$, which not only carry explicit physical meanings but also closely relate to oil-impregnated paper's insulation conditions. The quantitative insulation evaluation method based on these characteristic parameters requires further study.

\section{CONCLUSIONS}

As a summary, the behaviors of conduction and polarization processes in oil-impregnated paper samples in the frequency domain were ascertained to provide a base for devising a model for accurately describe the FDS test curve. A universal function model with specific physical meanings can provide several characteristic parameters that are related to moisture content or aging degree in oil-paper insulation. The following specific conclusions can be drawn:

a) Equation $-\mathrm{d} \varepsilon / \mathrm{d}\left(\log _{10} \omega\right)$ could be handled as an effective tool for separating the relaxation processes from its conduction processes in the low band of the FDS test curve. By this measure, the relaxation process is revealed in the lowfrequency band, which has been neither observed nor studied in the field of frequency spectrum analysis of oil-impregnated paper insulation. It remains as an untrodden field, for low frequency band has conventionally been regarded as a conduction-dominated range.

b) The generation mechanisms of dielectric processes in oilimpregnated paper samples under broadband are determined. The FDS test curve of this kind of insulation consists of a DC conduction process, a space charge polarization with a barrier height of $1.07 \mathrm{eV}$ in the low-frequency band, and interfacial polarization with a barrier height of $0.56 \mathrm{eV}$ in the relatively high-frequency band.

c) The application of separate fitting and direct recombining each dielectric response process, yields a function model of complete FDS test curve of oil-impregnated paper. In logarithmic coordinates, the conduction losses are linear with a slope of -1 , the space charge polarization obeys the Debye model and the interface polarization function is typically a H-N model. All the characteristic parameters in the proposed function model carry explicit physical meanings, and thus can be applied to the quantitative condition assessment in power equipment.

\section{ACKNOWLEDGMENT}

The authors thank the project supported by the National Key Research and Development Program of China (Grant No. 2017YFB0902705), and the National Natural Science Foundation of China (Grant No. 51507130 and 51777157). 